\title{
Aspirin may slow the progression of emphysema
}

The search for a factor other than smoking cessation that can mitigate lung function decline in chronic obstructive pulmonary disease (COPD) is ongoing, with disappointing results from interventions that have seemed theoretically promising, such as statins. A recent report from the MESA (Multi Ethnic Study of Atherosclerosis) Lung Study, ${ }^{[1]}$ examined another of these hypotheses: antiplatelet therapy.

Previous evidence has shown that platelets are elevated in COPD, and related to adverse outcomes. Platelet activation is increased in patients with stable COPD compared with age-matched healthy subjects and during an acute exacerbation compared with their stable condition. ${ }^{[2]}$ Platelet activation leads to microvascular constriction in the lung, which exacerbates damage by increasing lung neutrophils. In addition, platelet factor 4 and neutrophil elastase increase the incidence of COPD in animal models. Antiplatelet drugs have been shown to improve survival in patients with very severe oxygendependent COPD, and following hospital admission for an acute exacerbation. ${ }^{[2]}$ In a meta-analysis, antiplatelet therapy reduced allcause mortality in patients with COPD independent of the presence of ischaemic heart disease. ${ }^{[3]}$

The MESA Lung Study prospectively followed a cohort of 4257 patients from 6 communities in the USA who were free of cardiovascular disease, and of whom $54 \%$ were smokers. CT scan quantification of the percentage emphysema in the participants' lungs was performed at baseline and again after 10 years, and aspirin use during this time was documented and quantified. The main finding of the study was that aspirin use is associated with a $50 \%$ slower progression of CT emphysema ( $0.3 \% \mathrm{v} .0 .6 \%$ per year) - the magnitude was greater with regular aspirin use compared with irregular use. This outcome between aspirin use and emphysema progression was similar, regardless of the dose ( $81 \mathrm{mg}$ v. $300-325 \mathrm{mg})$.
Although aspirin was significantly associated with slower progression of CT emphysema, there was no such association with change in $\mathrm{FEV}_{1}$, although this is not an unexpected finding if one postulates a different pathogenesis of the two entities. Emphysema may be initiated by vascular endothelial damage (and therefore be responsive to platelet activation inhibition), while development of airflow obstruction (indicated by $\mathrm{FEV}_{1}$ reduction) could be an entirely separate process.

There were a number of technical factors complicating the imaging assessments over the 10-year course of the study, as well as the inevitable confounding factors and bias present in an observational study, which relies on self-reported use of medication. Despite this, the findings of this study should still inspire hope that in the future we may identify an intervention which can attenuate the tragic trajectory of COPD.

Alan Peter, MB BCh, FCP (SA), Cert Pulm

Chris Hani Baragwanath Academic Hospital, Johannesburg, South Africa alanpeter@ymail.com

Afr J Thoracic Crit Care Med 2018;24(3):123. DOI:10.7196/ SARJ.2018.v24i3.225

1. Aaron CP, Schwartz JE, Hoffman EA, et al. A Longitudinal cohort study of aspirin use and progression of emphysema-like lung characteristics on CT imaging: The MESA Lung Study. Chest 2018;154(1):41-50. https://doi.org/10.1016/j.chest.2017.11.031

2. Kirby M, Sin DD. Imaging end points in COPD clinical trials: Are we there yet? Chest 2018;154(1):3-5. https://doi.org/10.1016/j.chest.2018.01.050

3. Pavasini R, Biscaglia S, d'Ascenzo F, et al. Antiplatelet treatment reduces all-cause mortality in COPD patients: A systematic review and meta-analysis. COPD 2016;13(4):509-514. https://doi.org/10.3109/15412555.2015.1099620 